\title{
Photocatalytic Heteropolyacid/CdS (HPA/CdS) Composite for Degradation of Safranine T (ST)
}

\author{
Shuang Song ${ }^{a}$, Long Fan ${ }^{b}$, Shuyuan Xie ${ }^{c}$, Jinghai Liu ${ }^{d}$, Limei Duan ${ }^{\star}, e$
}

The College of Chemistry and Chemical Engineering, Inner Mongolia University for Nationalities, Tongliao, P. R. China 028000

a rascal_gentleman@sina.com, ${ }^{\mathrm{b}}$ jhliu2008@sinano.ac.c, ${ }^{\mathrm{c}}$ duanlmxie@126.com

\begin{abstract}
Keywords: CdS; heteropolyacid; composite material; Safranine T; photocatalysis
Abstract. A series of phosphotungstic acid and phosphomolybdic acid loaded CdS composites (HPW/CdS and HPMo/CdS) were prepared by impregnation method, and then characterized by FT-IR, XRD and TEM. The influences of different loading amounts on degradation rate of Safranine T under ultraviolet (UV) light irradiation were discussed. And, the changes of absorption peak and absorption intensity of Safranine T (ST) solution during the degradation process was monitored on UV-Vis spectrometer. The results show that synergistic effects exist in HPA/CdS and lead to improved photocatalytic degradation effects in comparison with corresponding CdS or heteropolyacid. HPMo/CdS with mass ratio of HPMo of $30 \mathrm{wt} \%$ exhibiting the best photocatalytic activity for degradation of ST under acid condition, with the degradation rate of $84.3 \%$ under UV light irradiation for $120 \mathrm{~min}$, and UV-Vis spectrum show that new substance is formed during the degradation process.
\end{abstract}

\section{Introduction}

Photocatalytic has much advantage, such as use sunlight as an energy source, let the chemical pollutants harmlessly degrade and no secondary pollutants generated after degradation, which made it a wide application prospect. The type of CdS - like semiconductor nanomaterials with so larger a light response range that make it a strong application feasibility and the research becoming deeper over time[1,2]. But the study also found that a single semiconductor nanomaterials is unconducive to the electronic - hole for a effective separation[3,4], because of its narrow forbidden bandwidth, recovery capability is so strong. And some of semiconductor materials, such as CdS , is so easy to reunite in the process of preparation that make its specific surface area decreased, quantum efficiency become lower and light corrosion effect are all severe impacts that unconducive to exhibit catalytic activity, which hinder the semiconductor nanomaterials are widely used in photocatalysis.

As a result, the preparation of new composite catalyst and then improving the performance of catalyst through synergy to expand the application scope, become a research hotspot.

Heteropolyacid compounds' structure are similar to that of metal oxide semiconductor, which has strong absorption in ultraviolet band, but because of the specific surface area is lesser, water-soluble is wonderful and hard to make a recycle utilization, which limits its application scope. Current research suggests that heteropolyacid (with the Keggin type)[5,6] /Fe3O4 magnetic catalysts for the degradation of methylene blue[7] and HPW/CdS support catalyst for the degradation of Rhodamine $\mathrm{B}[8]$, perform much better than a single component, recycling performance is also improved. The situation of the application of heteropolyacid/TiO2 has been comprehensively described by Hua-lan Zhou. The research on composite catalyst contained heteropolyacid is becoming more and more deeply.

In this paper, phosphotungstic acid and phosphomolybdic acid were supported on the CdS nanomaterials, and then, heteropolyacid /CdS composite catalyst were obtained. Safranine T was select as experimental object for photocatalytic degradation. A type of composite with higher catalytic activity performance by exploit their own advantages and produce synergistic effect, which are we expected. 


\section{Experimental}

Preparation of the composite of heteropolyacid/CdS. HPW/CdS and HPMo/CdS composites were prepared by impregnation method. A certain amount of HPMo or HPW was dispersed in distilled water under stirring in a beaker. Then the heteropolyacid solvent was obtained. Heteropolyacid solution and a certain amount of homemade CdS powder were mixed and stirring for 3 hours at $60^{\circ} \mathrm{C}$ to make it fully impregnated and then dried at $60^{\circ} \mathrm{C}$ for 6 hour. The number of composites catalyst were A-1, A-2, A-3 and A-4 when the HPMo composite amount were 15\%, $30 \%, 45 \%$ and $60 \%$. And when the HPW amount were 15\%, $30 \%$, $45 \%$ and $60 \%$ named B-1, B-2, B-3 and B-4.

Characterization. FTIR transmission spectra were performed on Nicolet 5700 IR spectrometer by using KBr pellets. XRD patterns were identified by AXS D8FOCUS X-ray powder diffractometer. TEM images were obtained on HITACHI H7600-TEM. Visible absorption spectra were measured on 722s (Shanghai precision \& scientific instrument Co., Ltd) visible light spectrophotometer.

Analysis of photocatalytic performance of composite. 50mg heteropolyacid /CdS composite powder was dispersed in $100 \mathrm{~mL}$ of Safranine $\mathrm{T}$ solution with a concentration of $20 \mathrm{mg} / \mathrm{L}$ in a photochemical reactor, then keep it in the dark for 20min under stirring to reach saturation absorption, adjusting the $\mathrm{pH}=4$ by adding HAc. The experiments were carried out after turn on the UV light placed $120 \mathrm{~mm}$ above the solution, keeping continuous stirring. Sampling every 20min, and take its supernatant fluid after centrifuged immediately. The absorbance value was determinated at 512nm, which is the maximum absorption wavelength of Safranine T solution, to determine the effect of photocatalyst for the degradation of dye.

\section{Results and Discussion}

Figure 1 shows that, the intense bands between 700 and $1100 \mathrm{~cm}^{-1}$ correspond to the characteristic peak of Keggin heteropolyacid, and there was no obvious change in absorpt peak position, the intensity of peaks increase go with the loadand of heteropolyacid in composite.
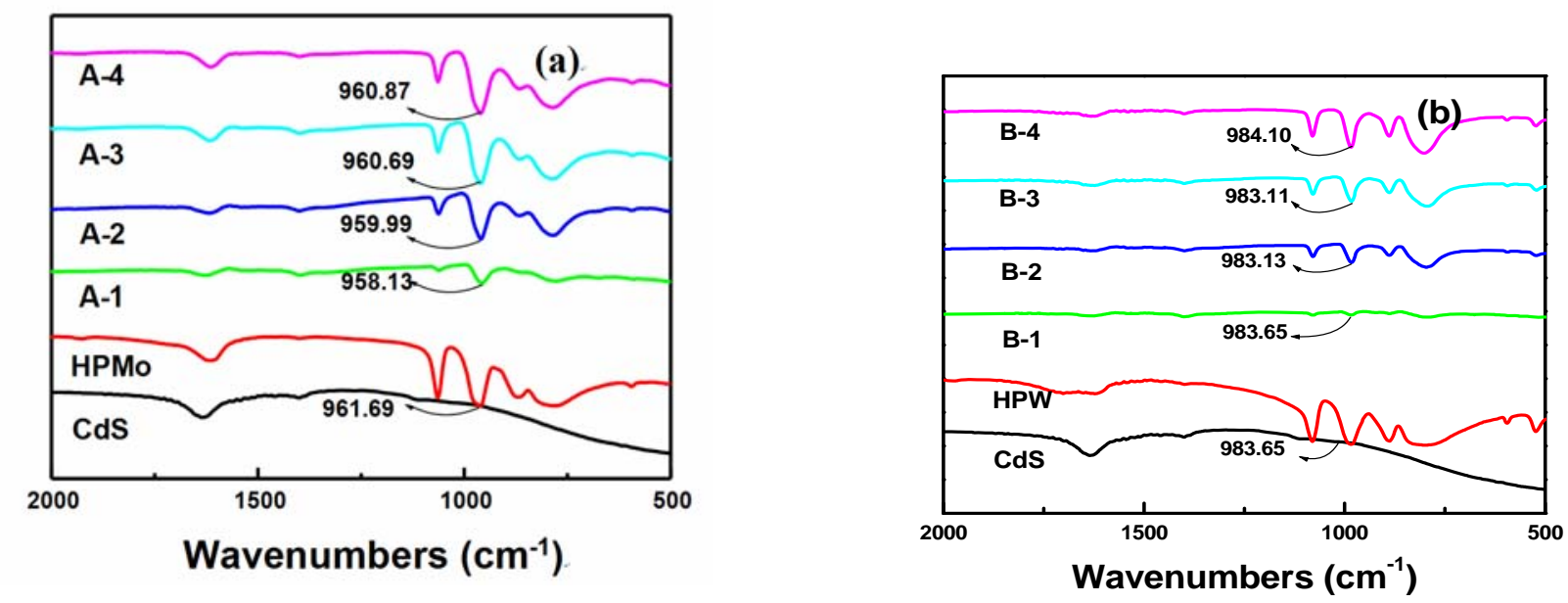

Fig.1 FT-IR of (a)CdS, HPMo and CdS/HPMo composites (AX),(b) CdS, HPW and CdS/HPW composites (BX)

The XRD pattern of CdS sample match the standard spectra well and the strong diffraction peak are the characteristic patterns of Keggin heteropolyacid structure. Only CdS characteristic diffraction peak could be observed when the mass ratio of heteropolyacid to CdS is 3 to 7 and 15 to 85 , Presumably, heteropolyacid was dispersed more evenly on the surface of CdS. While, the diffraction 
peak of heteropolyacid appeared but weakly when the ratio of heteropolyacid to CdS is 45 to 55 and 6 to 4, it could be determinded that heteropolyacid was not so evenly on the surface of CdS along with the increasely loadand.
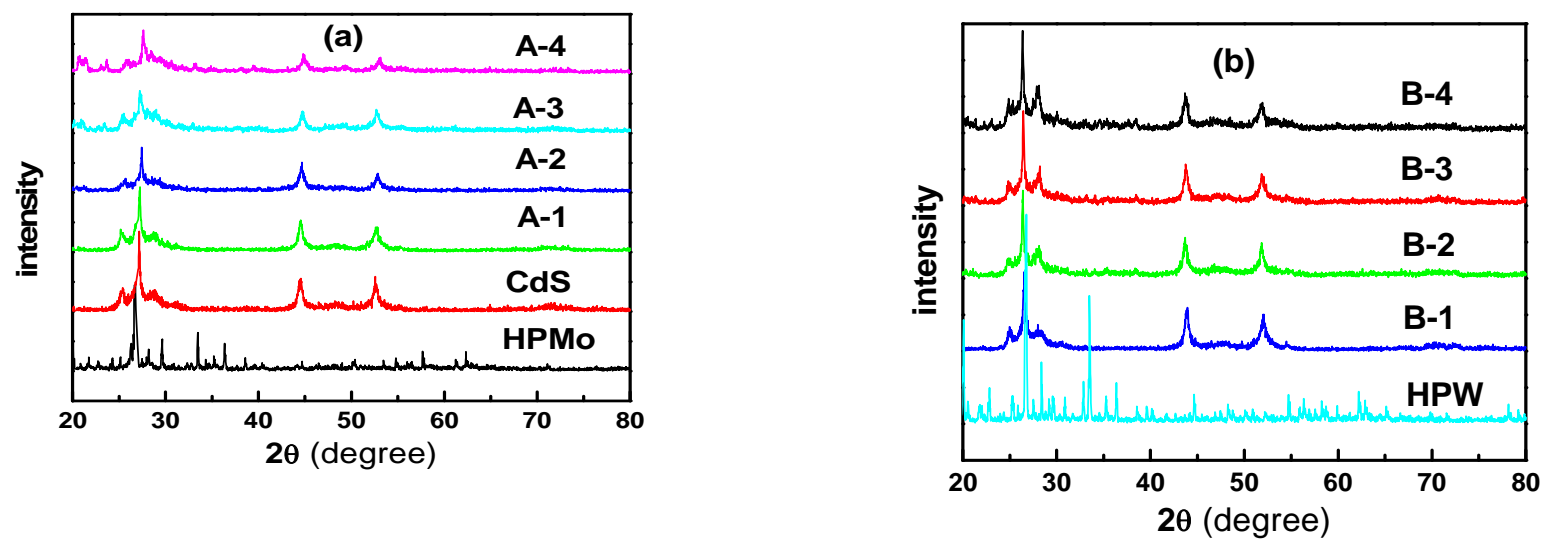

Fig.2 XRD patterns of (b) HPMo and CdS/HPMo composites (AX),(b) CdS, HPW and CdS/HPW composites (BX)
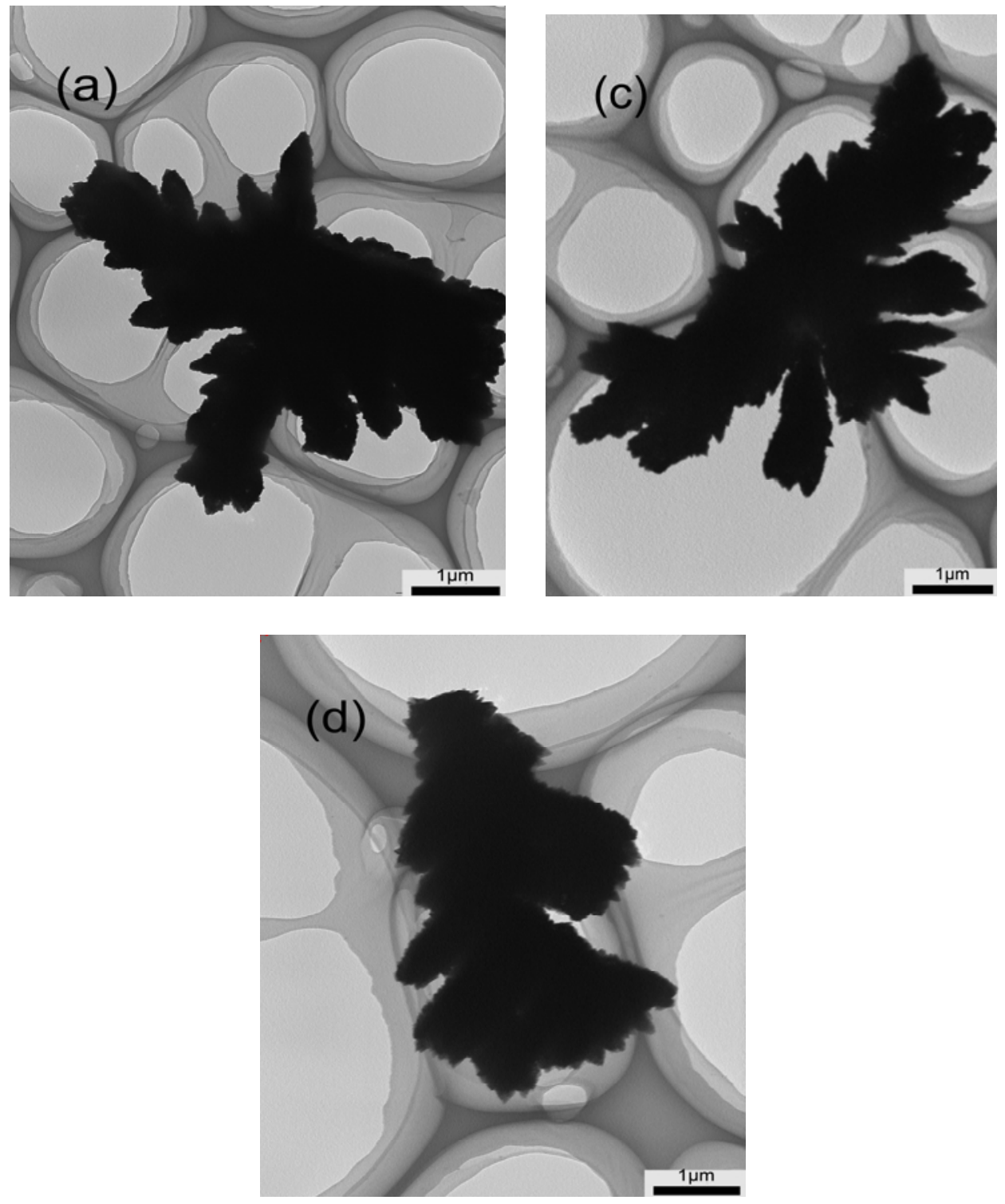

Fig.3 TEM images of (a)CdS (c)A-2(d)B-2 
TEM image in Fig.3 shows that the ginger-like morphology of (a) CdS and composite, there was no significant difference before and after the composite, (c) HPMo or (d) HPW nanoparticles are not easy to be observed.

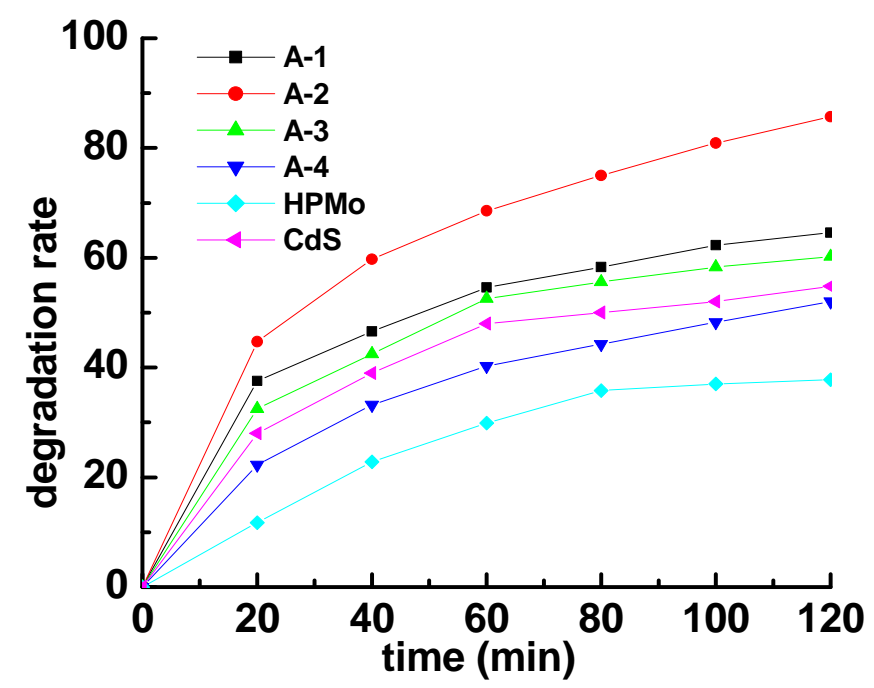

Fig. $4 \mathrm{pH}=4$, degradation efficiency of Safranine $\mathrm{T}$ with single CdS, single HPMo and different HPMo/CdS composites (AX) samples under UV light

It could be known from Fig.4, the degradation efficiency of Safranine T with composites A-1, A-2, A-3 is better than single CdS or HPMo. Too much or too less of heteropolyacid are both unconducive to increase the degradation activity of composite catalyst. Too less, synergistic effect is not obvious, while too much of heteropolyacid, degradation efficiency is influence by the decrease of CdS. A-2 (the ratio of HPMo to CdS is 3 to 7) exhibiting the best activity for degradation with the degradation rate of $84.3 \%$ for Safranine T under UV light.

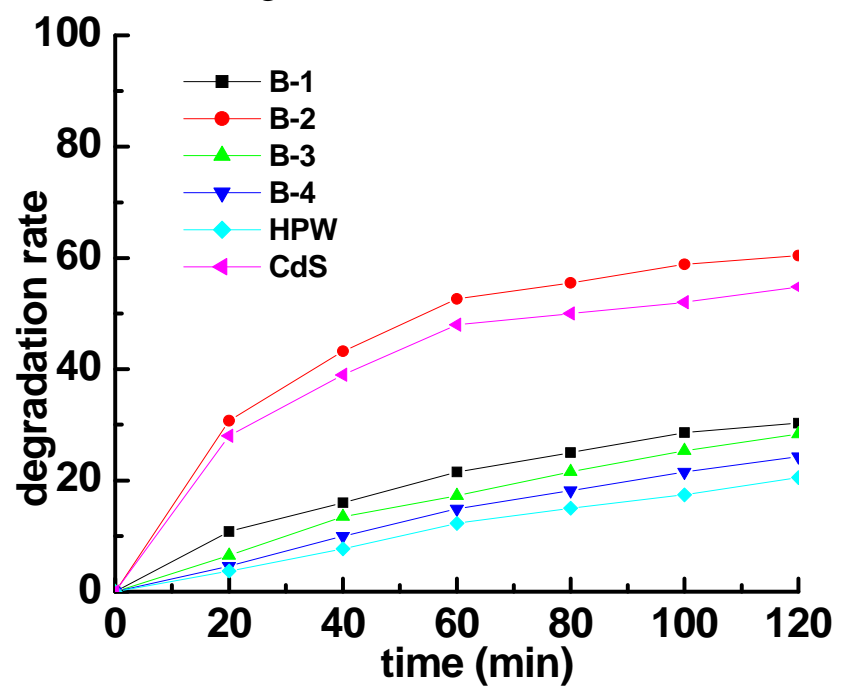

Fig. $5 \mathrm{pH}=4$, degradation efficiency of Safranine $\mathrm{T}$ with single CdS ,single HPW and different HPW/CdS composites (BX) samples under UV light

Figure 5 shows that degradation efficiency of Safranine T with HPW is not very well ,HPW exhibit so low degradation activity until compounded on CdS, but still lower than absolute CdS except B-2 (the ratio of HPW to CdS is 3 to 7). The reason why degradation efficiency pecking order are B-1 $>$ B-3 $>$ B-4 is the addition of CdS, the more, the better, and the synergy that desired is not appeared, or at least, not obvious. B-2 exhibits well on degradation efficiency of Safranine T because of synergy, higher than that of the CdS, but still not obviously. 
Compared with HPW/CdS, HPMo/CdS exhibit much better on degradation efficiency of Safranine T under UV light.

The changes of the absorbance value during the process of photocatalytic degradation of Safranine T with A-2 was examined, and then judging the situation degradation of Safranine T solution (initial concentration $10 \mathrm{mg} / \mathrm{L})$. $50 \mathrm{mg}$ A-2 catalyst was added to $100 \mathrm{~mL}$ Safranine $\mathrm{T}(20 \mathrm{mg} / \mathrm{L})$ solution, under stirring and keep the $\mathrm{pH}=4$, and degradation reaction started under UV light irradiation. Sampling every 20 min centrifugal separation, and then ultraviolet-visible absorption spectrum are determined and was shown in figure 6.

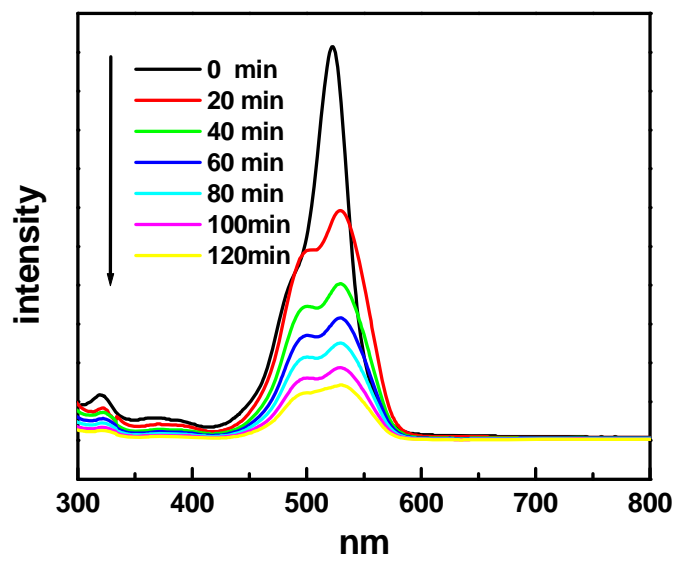

Fig.6 The chang of absorption of degradation process of Safranine T with B-2 under UV light

Figure 6 shows that absorption peak become more wide, Subtle but obviously,and new absorption peaks appeared during the process of the degradation of Safranine T. It suggests that Safranine T has been decomposed and the intermediate product appeared, but the absorption intensity in lowering.The reaction solution from red to reddish brown, and the absorbance of the solution is no longer in decline under UV light irradiation for $120 \mathrm{~min}$.

\section{Conclusions}

A series of HPMo/CdS and HPW/CdS with various compound ratio were prepared by impregnation method, the photocatalytic degradation efficiency of Safranine $\mathrm{T}$ under different conditions were examined. The results show that A-2 (the ratio of HPMo to CdS is 3 to 7 ) exhibit the best on the degradation of Safranine T in all samples. Varying degrees of synergy HPMo/CdS and HPW/CdS are observed when the ratio of composite is appropriate. And new substance is formed during the degradation process.

\section{Acknowledgments}

We acknowledge the financial supports of the National Natural Science Foundation of China (21461018 and 21303080.) and the Natural Science Fundation Committee of Inner Mongolia (2013MS0211 and 2013MS0216).

\section{References}

[1] A. Koistinen and M. Sillanpää. J. Nanosci. Nanotechnol. 11 (2011), p. 2090-2099

[2] Z. Y. Wang, Z. P. Yu, B. Liu, et al. Chem. Mater. Sci. 24 (2009), p. 698-701

[3] Zhang H, Zhu Y F. J Phys Chem C, 114(2010), p. 5822

[4] Zong X, Yan H J, Wu G P, Ma G J, Wen F Y, Wang L, Li C. J Am Chem Soc, 130(2008), p. 7176 
[5] Gagea B C, Lorgouilloux Y, Altinatas Y, et al. J. Catal, 265 (2009), p. 99-108

[6] Y. Yang, Q.Y. Wu, Y.H. Guo, et al. Journal of Molecular Catalysis A, 225 (2004) , p. 203- 212

[7] Bao Sharileaodu, Wang Tianshu, Liu Zongrui, et al. Preparation of heteropolyacid /Fe3O4 magnetic catalyst and its degradation behaviors for methylene blue solution.[J] Industril Catalysis, 2014, 22(10): 756-760. In Chinese

[8] T. S. Wang, S. Bao, L. Xu, et al. Applied Mechanics and Materials, 548(2014), p. 25-28 